\title{
Effects of Annealing Time on the Performance of OTFT on Glass with $\mathrm{ZrO}_{2}$ as Gate Dielectric
}

\author{
W. M. Tang, ${ }^{1,2}$ M. G. Helander, ${ }^{1}$ M. T. Greiner, ${ }^{1}$ Z. H. Lu, ${ }^{1,3}$ and W. T. Ng $^{2}$ \\ ${ }^{1}$ Department of Materials Science and Engineering, University of Toronto, 184 College Street, Toronto, ON, Canada M5S 3E4 \\ ${ }^{2}$ Department of Electrical and Computer Engineering, University of Toronto, 10 King's College Road, Toronto, ON, Canada M5S $3 G 4$ \\ ${ }^{3}$ Department of Physics, Yunnan University, 2 Cuihu Beilu, Yunnan, Kumming 650091, China
}

Correspondence should be addressed to W. M. Tang, wmtang@vrg.utoronto.ca

Received 15 July 2011; Accepted 6 October 2011

Academic Editor: Hsiao W. Zan

Copyright (c) 2012 W. M. Tang et al. This is an open access article distributed under the Creative Commons Attribution License, which permits unrestricted use, distribution, and reproduction in any medium, provided the original work is properly cited.

Copper phthalocyanine-based organic thin-film transistors (OTFTs) with zirconium oxide $\left(\mathrm{ZrO}_{2}\right)$ as gate dielectric have been fabricated on glass substrates. The gate dielectric is annealed in $\mathrm{N}_{2}$ at different durations $(5,15,40$, and 60 min) to investigate the effects of annealing time on the electrical properties of the OTFTs. Experimental results show that the longer the annealing time for the OTFT, the better the performance. Among the devices studied, OTFTs with gate dielectric annealed at $350^{\circ} \mathrm{C}$ in $\mathrm{N}_{2}$ for $60 \mathrm{~min}$ exhibit the best device performance. They have a small threshold voltage of $-0.58 \mathrm{~V}$, a low subthreshold slope of $0.8 \mathrm{~V} / \mathrm{dec}$ ade, and a low off-state current of $0.73 \mathrm{nA}$. These characteristics demonstrate that the fabricated device is suitable for low-voltage and low-power operations. When compared with the TFT samples annealed for $5 \mathrm{~min}$, the ones annealed for $60 \mathrm{~min}$ have $20 \%$ higher mobility and nearly two times smaller the subthreshold slope and off-state current. The extended annealing can effectively reduce the defects in the high-k film and produces a better insulator/organic interface. This results in lower amount of carrier scattering and larger CuPc grains for carrier transport.

\section{Introduction}

Owing to light weight, mechanical flexibility, and low-cost fabrication, organic thin-film transistors (OTFTs) have a wide range of applications such as sensors, flat-panel displays, and RFID tags [1-3]. The first OTFT based on organic semiconductor polythiophene was reported in 1986 [4]. Heavily doped silicon substrates are generally used for the fabrication of OTFT as they are highly conductive and can act as the gate of the devices. In addition, high-quality gate dielectric $\mathrm{SiO}_{2}$ can be thermally grown directly on the Si substrate. However, for the next generation of OTFTs and oxide TFTs fabricated on glass or plastic substrates, a metal gate electrode is necessary. By fabricating OTFTs on flexible plastic substrates, there is a potential in the future for producing roll-up displays that can be integrated into a small device such as a pen. There are three major types of dielectric used in OTFTs: inorganic dielectric, polymeric dielectric, and self-assemble layer. For inorganic dielectric materials, silicon dioxide is commonly used as the gate insulator in OTFT.
However, this kind of OTFT requires a relatively high voltage (about $100 \mathrm{~V}$ ) for operation. In order to reduce the operating voltage and hence the power consumption, high-k material is often used as a gate dielectric in OTFTs. Several high-k dielectrics have been employed to fabricate OTFT, for example, $\mathrm{HfO}_{2}$ [5], $\mathrm{Al}_{2} \mathrm{O}_{3}$ [6], $\mathrm{TiO}_{2}$ [7], $\mathrm{Ta}_{2} \mathrm{O}_{5}$ [8], and $\mathrm{BaTiO}_{3}[9]$. The performance of organic transistors depends largely on the quality of the gate insulator, the insulator/organic interface, the morphology of the organic thin film, and the charge injection process. It is essential to develop a suitable and high-quality gate insulator with appropriate morphology to achieve a smooth insulator/organic interface. The as-deposited high-k films are usually loosely packed and contain impurities and defects such as oxygen vacancies, oxygen interstitials, and/or oxygen deficiency [10]. These defects and impurities will cause transient charge trapping in the high-k dielectric and leakage current [11]. Various surface passivation methods have been developed in order to achieve high-quality high-k OTFTs, such as annealing in ultraviolet (UV) ozone and nitridation gases, surface 
treatment with octadecyltrichlorosilane (OTS), polymethylmethacrylate (PMMA), polyvinyl acetate and ion-beam irradiation, and using stack insulator structure. In this study, one of the most promising high-k dielectric for widespread application, zirconium oxide $\left(\mathrm{ZrO}_{2}\right)$, is used as the gate dielectric. $\mathrm{ZrO}_{2}$ is a stable metal oxide with a high dielectric constant $(\sim 15-25)$ [12] and a large band gap $(5.8 \mathrm{eV})$. It has been reported that $\mathrm{ZrO}_{2}$ has the lowest leakage current [13]. Moreover, it is a promising material for the fabrication of large-area flexible displays because the $\mathrm{ZrO}_{2}$ films can be transparent and have good adhesion with plastic substrates [14]. The effects of $\mathrm{ZrO}_{2}$ annealing treatment time in nitrogen ambient on the electrical properties of CuPc-based organic thin film transistors are investigated. The electrical and physical characteristics of the devices are presented.

\section{Experimental Details}

Corning 2947 glass substrates $\left(25 \times 25 \mathrm{~mm}^{2}\right)$ were used in this study as they are mechanical stable, low cost, and compatible with large area applications such as plasma televisions. The substrates were cleaned with a standard regiment of Alconox, acetone, methanol, and deionized water followed by UV ozone treatment for $15 \mathrm{~min}$. The substrates were then loaded into a sputtering chamber with a base pressure of $2.5 \times 10^{-6}$ Torr. A $150 \mathrm{~nm}$ thick $\mathrm{Al}$ gate was then deposited at room temperature with a deposition rate of $0.4 \AA / \mathrm{sec}$. A $40 \mathrm{~nm}$ thick $\mathrm{ZrO}_{2}$ was then deposited by sputtering from a zirconium target $(99.95 \%$ purity) with an $\mathrm{RF}$ power of $130 \mathrm{~W}$ in a mixed $\mathrm{Ar} / \mathrm{O}_{2}$ ambient $\left(\right.$ Ar to $\mathrm{O}_{2}$ ratio $\left.=4: 1\right)$. The chamber pressure during deposition was 5.67 mTorr. The samples then underwent annealing on a hotplate at $350^{\circ} \mathrm{C}$ in $\mathrm{N}_{2}$ for different durations ( $0 \mathrm{~min}, 5 \mathrm{~min}, 15 \mathrm{~min}$, $40 \mathrm{~min}$, and $60 \mathrm{~min}$ ). A layer of $40 \mathrm{~nm}$-type semiconductor, copper phthalocyanine $(\mathrm{CuPc})$ was then deposited by vacuum evaporation through a patterned shadow mask at room temperature. $\mathrm{CuPc}$ is a stable and promising $p$-type organic semiconductor with the major charge carriers as holes. It can be easily obtained in large quantity and high purity and hence particularly attractive for low-cost applications in dye processing, chemical sensors, and optical data storage $[15,16]$. The source and drain gold pads, $50 \mathrm{~nm}$ thick, were then deposited on top of the organic layer by thermal evaporation through a stainless steel mask with a channel length $L$ of $36 \mu \mathrm{m}$ and a channel width $W$ of $961 \mu \mathrm{m}$. The cross-section of the OTFT structure is as shown in Figure 1. The currentvoltage $(I-V)$ characteristics were measured by an HP 4155A semiconductor parameter analyzer. All the measurements were taken by a probe station at room temperature in the ambient atmosphere. An atomic force microscopy (Veeco Dimension 3100) in tapping mode was employed to analyze the surface morphology, grain size, and rms roughness of the high- $\mathrm{ZrO}_{2}$ and $\mathrm{CuPc}$ films.

\section{Results and Discussion}

Figure 2 shows the output characteristics of the OTFTs with $\mathrm{ZrO}_{2}$ film annealed for $0 \mathrm{~min}$ and $60 \mathrm{~min}$. Typical $I_{d}-V_{d}$ curves were obtained when a negative $V_{g}$ was applied to the
TABLE 1: Device parameters of the OTFTs.

\begin{tabular}{lccccc}
\hline & $0 \min$ & $5 \mathrm{~min}$ & $15 \mathrm{~min}$ & $40 \mathrm{~min}$ & $60 \mathrm{~min}$ \\
\hline$\mu\left(\times 10^{-3} \mathrm{~cm}^{2} / \mathrm{Vs}\right)$ & 1.19 & 1.21 & 1.23 & 1.31 & 1.43 \\
$S S(\mathrm{~V} /$ decade $)$ & 1.53 & 1.45 & 1.11 & 1.03 & 0.8 \\
$I_{\text {off }}(\mathrm{nA})$ & 2.59 & 1.73 & 1.39 & 0.76 & 0.73 \\
$N_{\max }\left(\times 10^{13} \mathrm{~cm}^{-2}\right)$ & 6.89 & 6.51 & 4.92 & 4.54 & 3.47 \\
\hline
\end{tabular}

devices. At a gate voltage of $-3 \mathrm{~V}$ and a drain voltage of $-4.5 \mathrm{~V}$, the device with $\mathrm{ZrO}_{2}$ film annealed for 0 min has a drain current of $77 \mathrm{nA}$, while the one annealed for $60 \mathrm{~min}$ has a larger drain current of $107 \mathrm{nA}$. The operating principle of OTFT is similar to that of traditional $p$-type MOSFET. The current flowed between the source and drain electrodes is modulated by the gate voltage $V_{g}$. The carrier mobility of the devices in the saturation regime can be calculated using the following equation:

$$
\mu=\frac{2 L\left(\partial I_{d}^{1 / 2} / \partial V_{g}\right)^{2}}{W C_{\mathrm{ox}}},
$$

where $C_{\mathrm{ox}}$ is the unit capacitance of the insulator and $\mu$ is the carrier mobility. Figure 3 shows the transfer characteristics of $I_{d}$ versus $V_{g}$ at a fixed $V_{d}$ of $-2.5 \mathrm{~V}$ for the devices. The subthreshold slope, $S S$, is a very important parameter for OTFTs as it can be used to evaluate the switching characteristics of the OTFTs. SS is defined as $\partial V_{g} / \partial \log _{10}\left(I_{d}\right)$ evaluated at the steepest slope of the plot. From SS, the maximum density of the surface states $N_{\max }$ at the organic semiconductor/dielectric interface can be estimated as

$$
N_{\max }=\left[\frac{S S \log (e)}{(k T / q)}-1\right] \frac{C_{\mathrm{o}}}{q},
$$

where $q$ is the electronic charge, $k$ the Boltzmann constant, and $T$ the temperature in Kelvin.

The important parameters of the devices extracted from the transistor characteristics are summarized in Table 1. It is found that the performances of the OTFTs including mobility, subthreshold slope, surface states density, and off-state current $I_{\text {off }}$ improve with annealing duration. This should be due to the fact that the OTFT annealed for longer duration can have a denser $\mathrm{ZrO}_{2}$ film and a thicker interfacial layer $[17,18]$ with lower interface-trap density to suppress the leakage associated with high-k materials. In addition, longer annealing can remove more deep traps, oxide charges, and unsaturated bonds in the $\mathrm{ZrO}_{2}$ dielectric and thus lead to a significant reduction of $S, N_{\max }$ and $I_{\text {off }}$. It has been reported that dielectric roughness can affect the performance of OTFT $[19,20]$. Figure 4 shows the AFM images of the $\mathrm{ZrO}_{2}$ films with different annealing times. It is found that extending the annealing time from $0 \mathrm{~min}$ to $60 \mathrm{~min}$ can reduce the gate-dielectric surface roughness (by $9 \%$ as measured using AFM). This can contribute to the reduction of trap states, interface defects, and surface scattering on charge carriers leading to higher mobility and smaller subthreshold slope. A smoother insulator surface is also more favorable for the growth of better quality organic film resulting in larger grains 


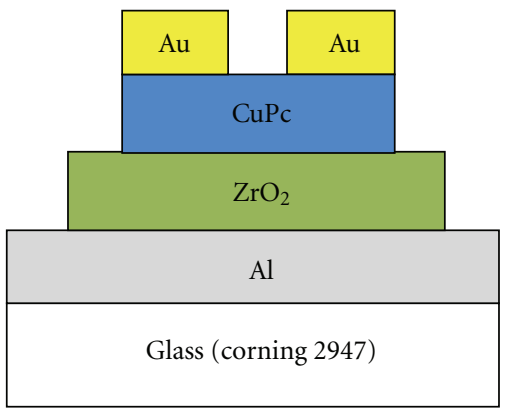

Figure 1: Schematic diagram of a CuPc transistor.

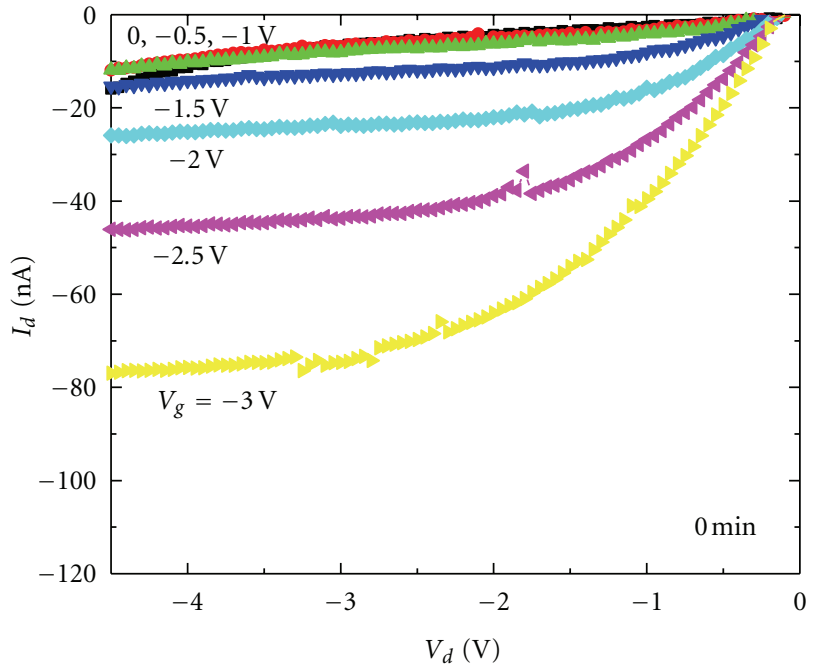

(a)

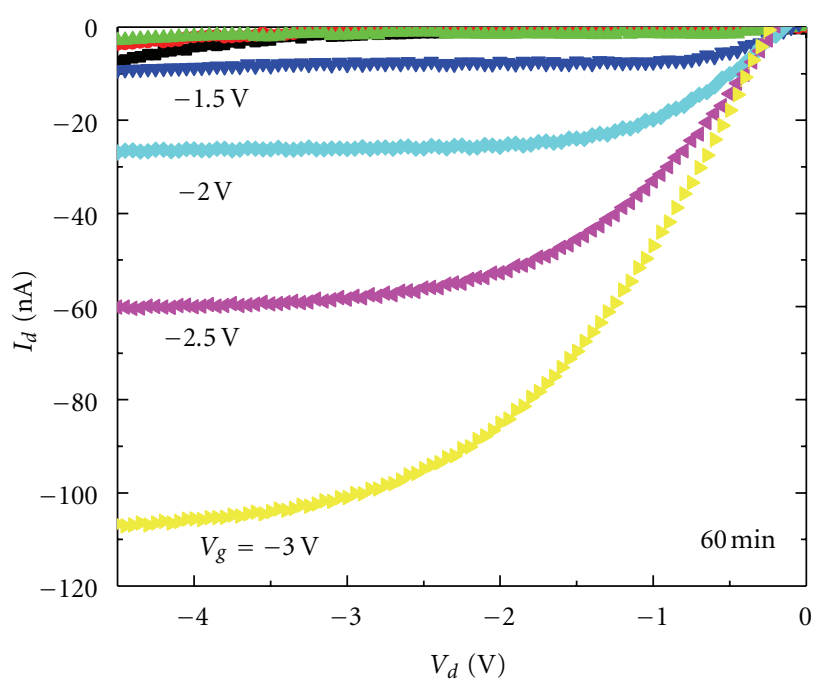

(b)

FIGURE 2: Output characteristic curves of OTFTs with $\mathrm{ZrO}_{2}$ gate dielectric annealed in $\mathrm{N}_{2}$ for (a) 0 min and (b) $60 \mathrm{~min}$.

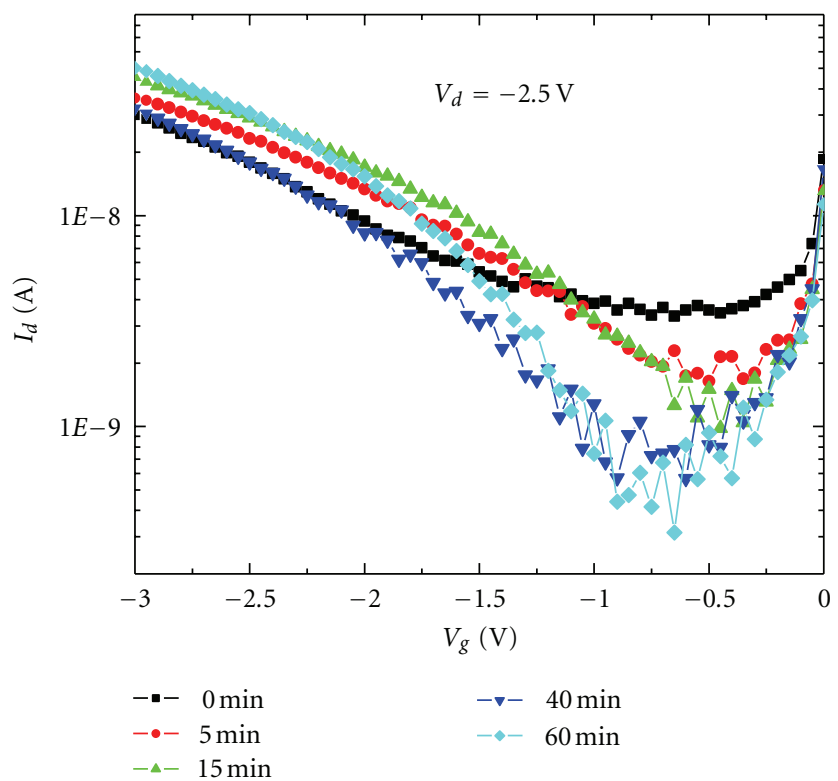

FIgURE 3: Transfer characteristic curves of OTFTs with $\mathrm{ZrO}_{2}$ gate dielectric annealed for various durations. 


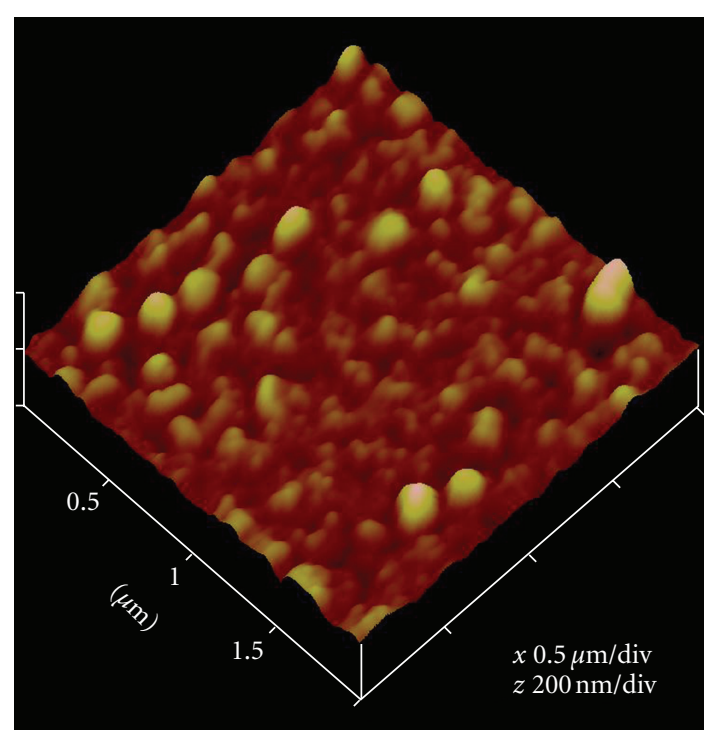

(a)

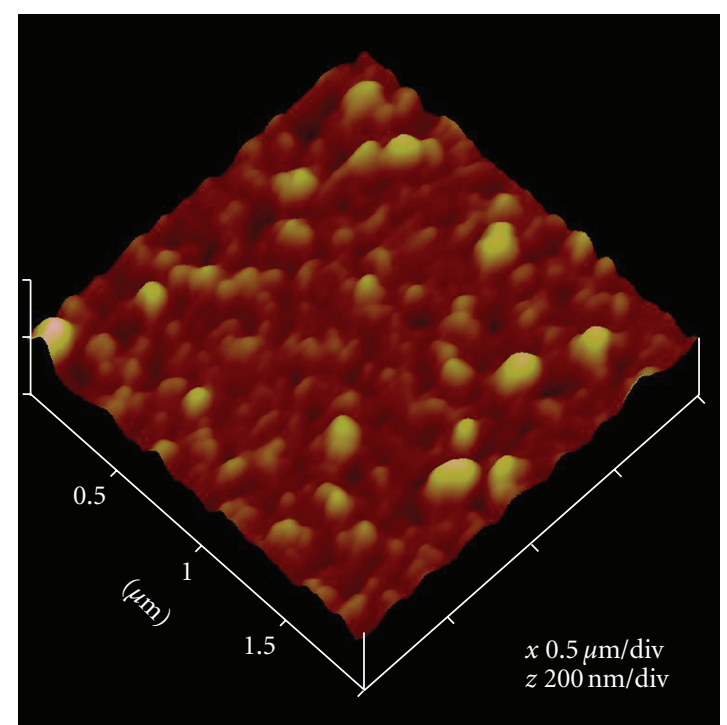

(b)

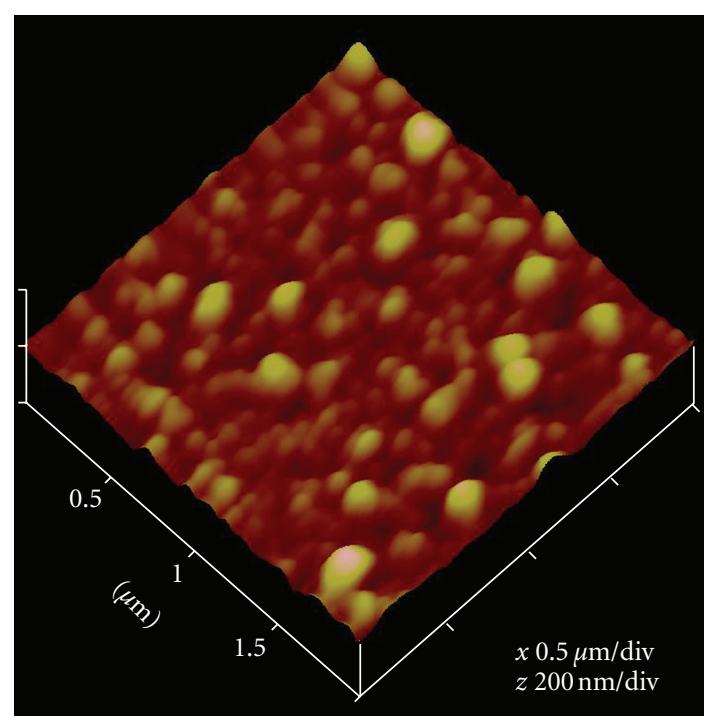

(c)

Figure 4: AFM images (scan area of $2 \mu \mathrm{m} \times 2 \mu \mathrm{m}$ ) of $\mathrm{ZrO}_{2}$ surface with different annealing treatment times: (a) 0 min, (b) 15 min, and (c) $60 \mathrm{~min}$.

$\mathrm{CuPc}$ film grown on the surface of $\mathrm{ZrO}_{2}$ ( $\sim 9 \%$ larger). This reduces the grain boundaries in the conduction channel for higher mobility of the device [21,22].

\section{Conclusions}

In summary, CuPc-based OTFT on glass substrate with high$\mathrm{k} \mathrm{ZrO}_{2}$ as gate insulator has been fabricated and studied. The effects of annealing time on the electrical properties of the devices are investigated. The study has demonstrated that $\mathrm{ZrO}_{2}$ is a promising gate dielectric material for obtaining low operating voltage and low power consumption. Experimental results show that the OTFTs with $\mathrm{ZrO}_{2}$ gate-dielectric annealed for $60 \mathrm{~min}$ have 20\% higher mobility, 50\% smaller subthreshold slope, $72 \%$ lower off-state current and 50\% smaller maximum density of surface states than the one annealed for $0 \mathrm{~min}$. The possible reasons are that longer annealing time can enhance the densification of the $\mathrm{ZrO}_{2}$ film, the dielectric properties of the $\mathrm{ZrO}_{2}$ film, and the quality of the $\mathrm{CuPc} / \mathrm{ZrO}_{2}$ interface, resulting in less carrier scattering and better grain growth.

\section{Acknowledgment}

The authors would like to acknowledge funding for this research from the Natural Sciences and Engineering Research Council (NSERC) of Canada, and Science and Technology Promotion Project of Yunnan Province (Grant nos. 2009 CI130 and 2007A0017z). 


\section{References}

[1] P. Mach, S. J. Rodriguez, R. Nortrup, P. Wiltzius, and J. A. Rogers, "Monolithically integrated, flexible display of polymer-dispersed liquid crystal driven by rubber-stamped organic thin-film transistors," Applied Physics Letters, vol. 78, no. 23, pp. 3592-3594, 2001.

[2] M. Halik, H. Klauk, U. Zschieschang et al., "Low-voltage organic transistors with an amorphous molecular gate dielectric," Nature, vol. 431, no. 7011, pp. 963-966, 2004.

[3] T. Someya and T. Sakurai, "Integration of organic field-effect transistors and rubbery pressure sensors for artificial skin applications," in Proceedings of the IEEE International Electron Devices Meeting, pp. 203-206, December 2003.

[4] A. Tsumura, H. Koezuka, and T. Ando, "Macromolecular electronic device: field-effect transistor with a polythiophene thin film," Applied Physics Letters, vol. 49, no. 18, pp. 1210-1212, 1986.

[5] J. Tardy, M. Erouel, A. L. Deman et al., "Organic thin film transistors with $\mathrm{HfO}_{2}$ high-k gate dielectric grown by anodic oxidation or deposited by sol-gel," Microelectronics Reliability, vol. 47, no. 2-3, pp. 372-377, 2007.

[6] J. B. Koo, J. W. Lim, S. H. Kim et al., "Pentacene thin-film transistors and inverters with plasma-enhanced atomic-layerdeposited $\mathrm{Al}_{2} \mathrm{O}_{3}$ gate dielectric," Thin Solid Films, vol. 515, no. 5, pp. 3132-3137, 2007.

[7] G. Wang, D. Moses, A. J. Heeger, H. M. Zhang, M. Narasimhan, and R. E. Demaray, "Poly(3-hexylthiophene) field-effect transistors with high dielectric constant gate insulator," Journal of Applied Physics, vol. 95, no. 1, pp. 316-322, 2004.

[8] C. Bartic, H. Jansen, A. Campitelli, and S. Borghs, " $\mathrm{Ta}_{2} \mathrm{O}_{5}$ as gate dielectric material for low-voltage organic thin-film transistors," Organic Electronics, vol. 3, no. 2, pp. 65-72, 2002.

[9] N. Hiroshiba, R. Kumashiro, K. Tanigaki et al., "Rubrene single crystal field-effect transistor with epitaxial $\mathrm{BaTiO}_{3}$ high- $\mathrm{k}$ gate insulator," Applied Physics Letters, vol. 89, no. 15, Article ID 152110, 2006.

[10] R. K. Nahar, V. Singh, and A. Sharma, "Study of electrical and microstructure properties of high dielectric hafnium oxide thin film for MOS devices," Journal of Materials Science, vol. 18, no. 6, pp. 615-619, 2007.

[11] B. H. Lee, S. C. Song, R. Choi, and P. Kirsch, "Metal electrode/high- $\kappa$ dielectric gate-stack technology for power management," IEEE Transactions on Electron Devices, vol. 55, no. 1, pp. 8-20, 2008.

[12] C. C. Fulton, T. E. Cook, G. Lucovsky, and R. J. Nemanich, "Interface instabilities and electronic properties of $\mathrm{ZrO}_{2}$ on silicon (100)," Journal of Applied Physics, vol. 96, no. 5, pp. 2665-2673, 2004

[13] W. J. Qi, R. Nieh, B. H. Lee et al., "MOSCAP and MOSFET characteristics using $\mathrm{ZrO}_{2}$ gate dielectric deposited directly on Si," in Proceedings of the 1999 IEEE International Devices Meeting (IEDM), pp. 145-148, December 1999.

[14] W. J. Qi, R. Nieh, B. H. Lee et al., "Performance of MOSFETs with ultra thin $\mathrm{ZrO}_{2}$ and $\mathrm{Zr}$ silicate gate dielectrics," in Proceedings of the 2000 Symposium on VLSI Technology, pp. 4041, June 2000.

[15] H. E. Katz, L. Torsi, and A. Dodabalapur, "Synthesis, material properties, and transistor performance of highly pure thiophene oligomers," Chemistry of Materials, vol. 7, no. 12, pp. 2235-2237, 1995.

[16] H. E. Katz, A. Dodabalapur, L. Torsi, and D. Elder, "Precursor synthesis, coupling, and TFT evaluation of end-substituted thiophene hexamers," Chemistry of Materials, vol. 7, no. 12, pp. 2238-2240, 1995.

[17] R. Jiang, E. Q. Xie, and Z. F. Wang, "Effect of inner oxygen on the interfacial layer formation for $\mathrm{HfO}_{2}$ gate dielectric," Journal of Materials Science, vol. 42, no. 17, pp. 7343-7347, 2007.

[18] L. Pereira, P. Barquinha, E. Fortunato, and R. Martins, "Low temperature processed hafnium oxide: structural and electrical properties," Materials Science in Semiconductor Processing, vol. 9, no. 6, pp. 1125-1132, 2006.

[19] D. Knipp, R. A. Street, A. Völkel, and J. Ho, "Pentacene thin film transistors on inorganic dielectrics: morphology, structural properties, and electronic transport," Journal of Applied Physics, vol. 93, no. 1, pp. 347-355, 2003.

[20] S. Steudel, S. De Vusser, S. De Jonge et al., "Influence of the dielectric roughness on the performance of pentacene transistors," Applied Physics Letters, vol. 85, no. 19, pp. 4400-4402, 2004.

[21] M. C. Kwan, K. H. Cheng, P. T. Lai, and C. M. Che, "Improved carrier mobility for pentacene TFT by $\mathrm{NH}_{3}$ annealing of gate dielectric," Solid-State Electronics, vol. 51, no. 1, pp. 77-80, 2007.

[22] K. H. Cheng, W. M. Tang, L. F. Deng, C. H. Leung, P. T. Lai, and C. M. Che, "Correlation between carrier mobility of pentacene thin-film transistor and surface passivation of its gate dielectric," Journal of Applied Physics, vol. 104, no. 11, Article ID 116107, 2008. 

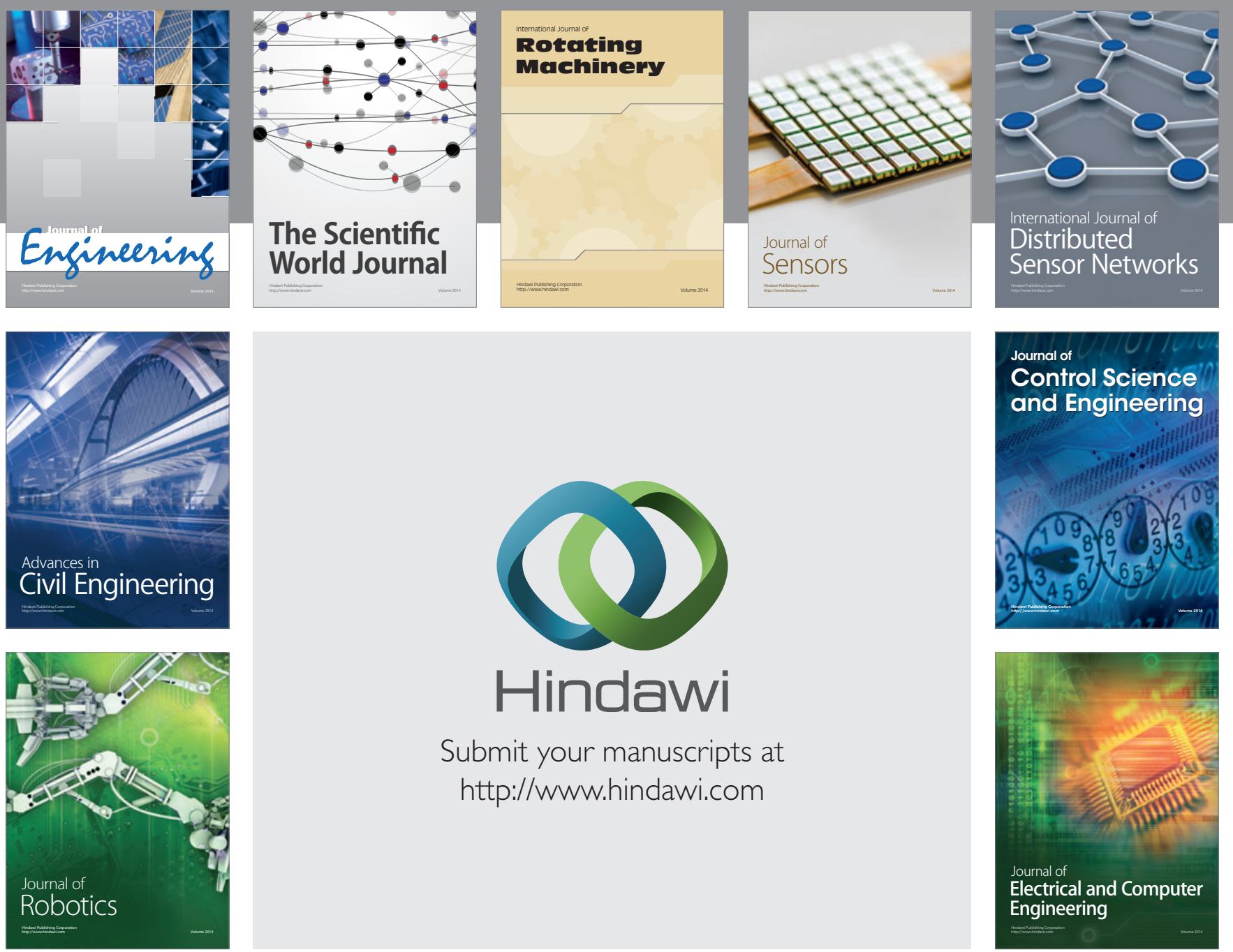

Submit your manuscripts at

http://www.hindawi.com
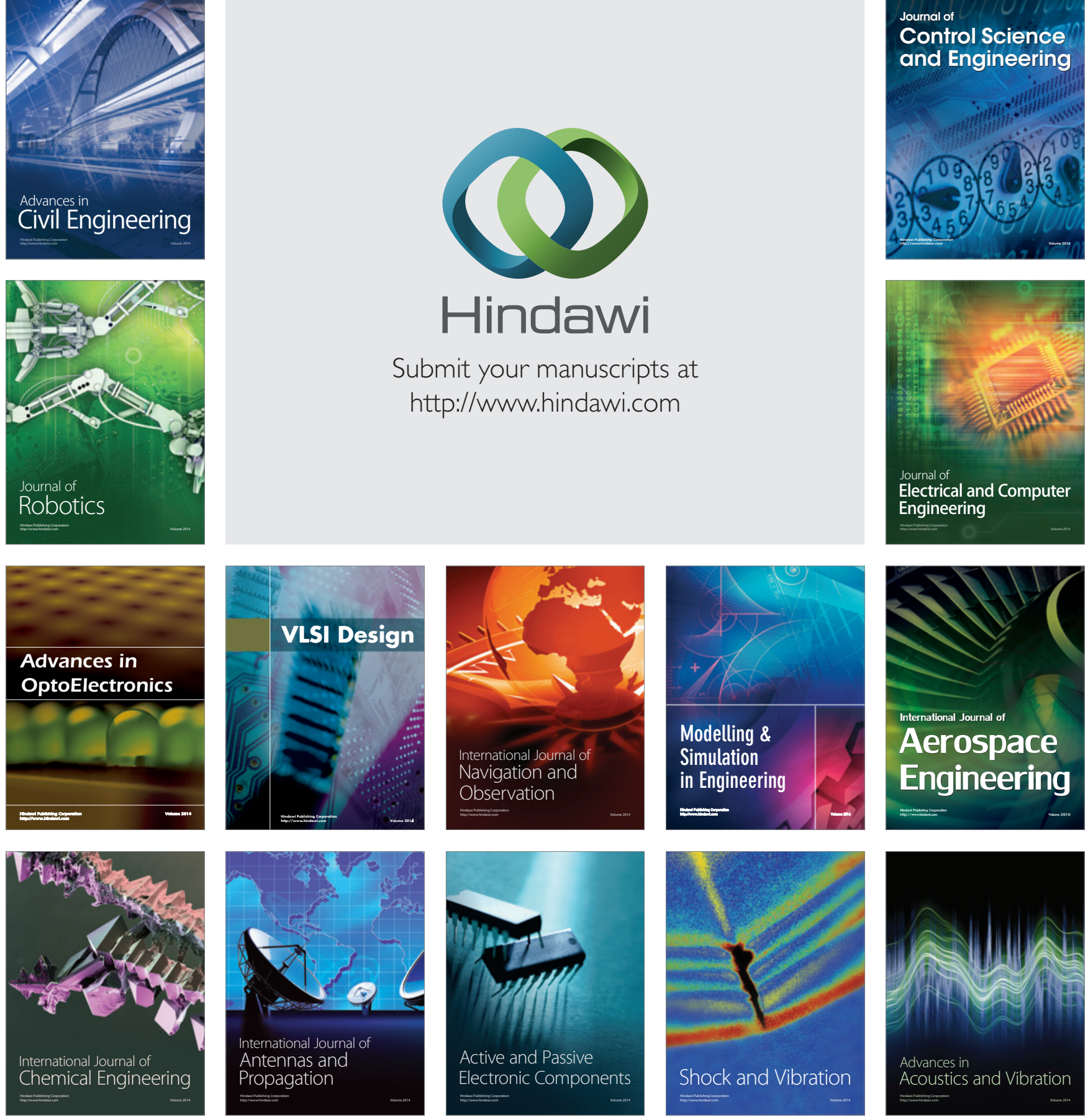